\title{
Optimization of Induced Velocity for Plasma Actuator with Multiple Encapsulated Electrodes using Response Surface Methodology
}

\author{
Rasool Erfani, ${ }^{*}$ Tohid Erfani, ${ }^{\dagger}$ Craig Hale, ${ }^{\ddagger}$ \\ Konstantinos Kontis ${ }^{\S}$ and Sergei V.Utyuzhnikov $~$ \\ The University of Manchester, Aero-Physics and Advance Measurement Technology Laboratory, \\ Sackville Street, Manchester, M60 1QD, Great Britain
}

\begin{abstract}
In design problem such as a new configuration of plasma actuator for maximizing the velocity of the airflow, experimental setup is done by an ad-hoc procedure. This provides the researcher with a relationship of the input parameters (width of the electrode, distance of the electrodes, the voltage and etc) and the velocity. As the experiments are time consuming and expensive in most of the cases, the above method is not always a reasonable approach in finding the optimal plasma configuration. In this paper response surface methodology, a surrogate modelling approach, is used to allow a systematic investigation in setting the experiments and finding the optimal plasma configuration. This allows the researcher to consider the uncertainty in observation and find a reliable approximate model for the induced velocity. Furthermore, the velocity of the airflow is modelled with small while enough number of experimental setups. The model is validated with the experimental data.
\end{abstract}

\section{Introduction and motivation}

$\mathrm{D}$ URING the past decade in the field of flow control, the use of plasma actuators has been implemented by many researchers. This new approach involves the use of dielectric barrier discharge (DBD) plasma which can be produced by means of two asymmetric electrodes separated by dielectric material. One of the electrodes is typically exposed to the air and connected to the high voltage supply while the other one is fully covered by the dielectric material and is earthed. Having no moving parts in its configuration, near instantaneous response, relatively low power consumption in addition to a wide range of operational frequencies made this technique non-negligible in flow control field. They have shown their ability to manipulate boundary layer, ${ }^{1-5}$ delay separation on airfoils ${ }^{6}$ and turbine blades, ${ }^{7}$ the laminar to turbulent transition point manipulation, ${ }^{8}$ control of separation on the stationary ${ }^{9}$ and oscillating airfoils,${ }^{10}$ wake of cylinder modification ${ }^{11-14}$ leading to reduced noise levels. ${ }^{15}$ Enloe et al. and Roth et al. presented numerous findings on the physics of plasma actuators, geometric arrangements based on measurements of thrust output, voltage and plasma emission measurements. ${ }^{16-22}$ A new design of plasma actuator developed at the University of Manchester has been shown to increase the induced velocity using multiple encapsulated electrodes. ${ }^{23-25}$

In most of the studies, the effect of one parameter on velocity is being studied while the others are kept constant at a prescribed value. This ad-hoc process continues for all the parameters to finally find a relationship between velocity and different type of actuator configuration.

Although this methodology provides the best configuration amongst the implemented experiments, there is no guarantee for finding the best possible configuration. That is, the final configuration is only limited to the already implemented setups which may not be optimum globally.

\footnotetext{
*Post Graduate Researcher, AIAA Student Member, Email: rasool.erfani@postgrad.manchester.ac.uk

${ }^{\dagger}$ Post Graduate Researcher, AIAA Student Member

${ }^{\ddagger}$ Post Graduate Researcher, AIAA Student Member

§Professor, School of MACE, AFAIAA

『Senior Research Fellow, School of MACE
} 
It also should be taken into account that each experiment is an expensive job to be done. In addition, there may be many redundant experiments without any use which only adds the cost of simulation. Furthermore, this method is exhaustive when the number of parameters (input variables) is large. Add to this difficulty is the investigation of the true value of velocity in presence of the human uncertainty and error in recording the observations. These lead to seeking a more systematic way for finding the effects of each parameter on the velocity. The methodology should be able to set the most informative set of experiments while keeping the number of experiments as low as possible. Finally, one may expect to find a new configuration of plasma actuator, which uses multiple encapsulated electrodes, more optimally.

To do so, we will use surrogate modelling. ${ }^{26}$ Surrogate modelling alleviating the above burdens by constructing approximation models. These methods effectively follow the behaviour of the simulation results as closely as possible. Surrogate modelling includes response surface methodology, ${ }^{27,}{ }^{28}$ Neural networks, ${ }^{29}$ evolutionary programming ${ }^{30}$ and Kriging interpolation. ${ }^{31,32}$ Examples of widely used surrogate modelling includes the multidisciplinary design of the high speed civil transport, ${ }^{33,34}$ structural optimization, ${ }^{35}$ blade shape design optimization ${ }^{36}$ and Aerospike Nozzle Design. ${ }^{37}$

In this study, we chose RSM to approximate a mathematical function for the velocity of the airflow in presence of the width of the electrode, voltage and the distance of the upper and lower electrode as design variables. A numerical optimization is carried out to find the maximization of the velocity with the optimum value of the design variables.

The paper is organized as follow. Section 1 gives the overview of the problem and optimization formulation. In Section 2 and 3 RSM and design of experiments are illustrated. The results and analysis are discussed in section 4 while in section 5 we conclude the paper and give recommendation for future work.

\section{Problem definition}

As mentioned before, the purpose of this paper is to present a systematic methodology in order to find the best possible configuration of plasma actuator in order to maximize the induced velocity of the airflow. The new configuration of plasma actuator uses multiple encapsulated electrodes. A sketch of the electrode can be seen in Figure 1. Plasma actuator consists of one exposed electrode which defines the baseline and three more electrodes positioned under it and isolated by Kapton material as a dielectric. In Figure 1, the different parameters of the problem are defined. Obviously finding the best possible configuration by changing these parameters as well as voltage and frequency is an exhaustive job. In the following section we discuss this issue and introduce an optimization methodology to do so.
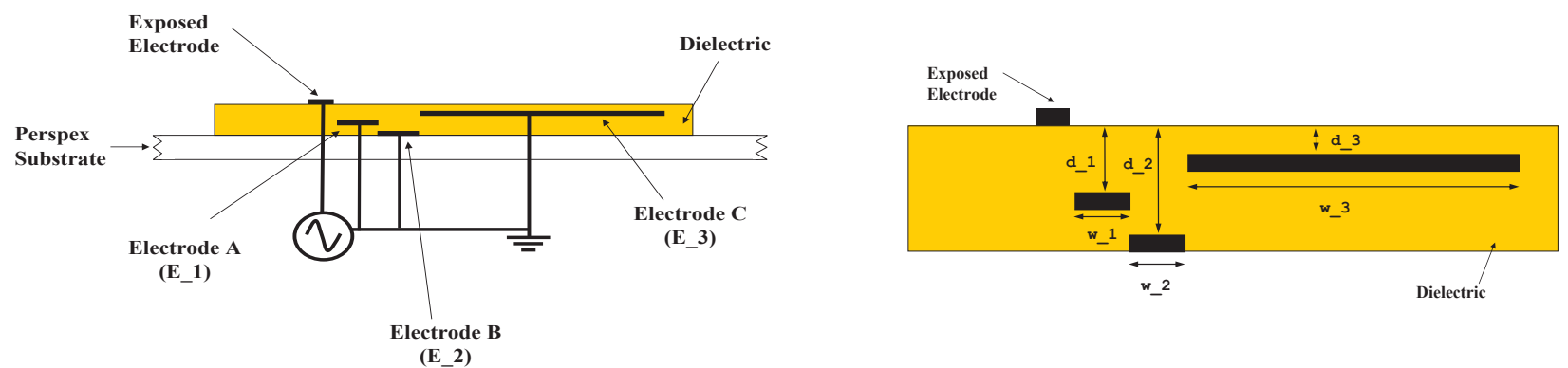

Figure 1: Multiple-Encapsulated plasma actuator (left), design parameters of the actuator (right)

\section{A. Optimization problem formulation}

Obviously, the above defined problem is a optimization task, which should be formulated subject to some geometrical constraints; the width of the electrodes, the voltage, frequency and the distance of the electrode from the exposed one (See Figure 1).

The induced velocity is the desired measure for maximization. Therefore, it is selected as the objective function for this optimization problem. The following is the optimization problem formulated for our purpose: 


$$
\begin{aligned}
\text { Maximize } & \text { Velocity, } \\
\text { s.t. } & 180 \leq d_{1}, d_{3} \leq 540 \\
& 5 \leq w_{1}, w_{3} \leq 40 \\
& 10 \leq \text { vol } \leq 16, \\
& 500 \leq \text { freq } \leq 20000, \\
& d_{2}=360, w_{2}=5,
\end{aligned}
$$

where parameters are listed in Table 1.

\begin{tabular}{ccccccc}
\hline & \multicolumn{5}{c}{ Design variables } \\
\cline { 2 - 7 } Name & voltage & Frequency & depth of $E_{1}$ & depth of $E_{3}$ & width of $E_{1}$ & width of $E_{3}$ \\
\hline symbol & vol & freq & $d_{1}$ & $d_{3}$ & $w_{1}$ & $w_{3}$ \\
unit & $\mathrm{V}$ & $\mathrm{Hz}$ & $\mathrm{mm}$ & $\mathrm{mm}$ & $\mathrm{mm}$ & $\mathrm{mm}$ \\
\hline
\end{tabular}

Table 1: Design variables

To solve the above problem the velocity should be formalized and an algorithm should be used for finding the solution. For estimating the velocity we use surrogate modelling technique. MATLAB is used for solving the above problem for performing the sequential quadratic programming (SQP).

\section{Response Surface Modeling Method}

Response surface methodology (RSM) is an approximation technique for analyzing the experimental data in order to construct a model for the behaviour of the system. The advantage of using the RSM is on its capability in situation in which there are inevitable noise. In addition, RSM can be applied for modelling where there are few experiments. This is of the most interest as the experimental setups are both time consuming and expensive to be done.

RSM performs a series of experiments for a predefined set of design points (independent variables) and using the responses (observations) obtained, it constructs a surface over the design space. For obtaining such a surface, RSM implements the regression analysis.

In many RSM applications, the quadratic polynomial is employed as the prescribed model. The reason lies in the fact that cubic and higher order polynomial need huge experiments which makes the RSM expensive. In addition, cubic and higher order polynomial generates the local minimum/maximum in the response surface. This abandons some optimizers to obtain the global optimum point in optimization process. Therefore, for having an accurate model while keeping the computational expense lower, the quadratic polynomial is used. The response surface, then, is expressed as follows:

$$
F=\text { velocity }=b_{0}+\sum_{j} b_{j} x_{j}+\sum_{j} \sum_{i} b_{i j} x_{i} x_{j}
$$

where $F$ is the response function, $x_{i}$ and $x_{j}$ are the design variables, and $b_{i}, b_{j}, b_{i j}$ are the unknown polynomial coefficients which are to be determined through regression analysis.

For the purpose of this paper, the response is the velocity of the airflow and the design variables are the ones listed in Table 1 (See Figure 1)

To fit the above model we need to set the most informative experiments for combinations of the prescribed design variables to record the velocity (response) for each of them. Design of experiments is employed for this purpose. 


\section{Design of Experiments Theory}

Building an approximation model involves choosing the most revealing sample of data representing the whole design space. Design of Experiments $(\mathrm{DoE})^{28}$ is a method for selecting the input parameter values as an appropriate sample at which a limited number of experiments to be conducted for recording the response value.

In literature, variety of alternatives in DoE exists. These include Full Factorial Designs, Fractional Factorial Designs, Plackett-Burman designs, Central-Composite Designs, Taguchi designs, D-Optimal designs and etc. ${ }^{27}$ All of these methods have some pros and cons. Therefore, the most relevant one should be implemented based on the problem under the study. The choice of the design of experiments has a large influence on the accuracy of the approximation and the cost of constructing the response surface.

For our purpose, D-Optimal Experimental Designs is chosen, because of its favourite properties. It requires fewer number of experimental runs and simulations compared to the other methodologies. In addition, it can be used for irregular shaped design space which is the case for our study.

\section{A. D-Optimal Experimental Designs}

In D-optimal design a sample of experiments in a design space is chosen from a larger set of candidate points. The objective is to minimize the variance (uncertainty) in the estimated coefficients of the polynomial model (1). The optimization methods are used to create D-optimal experimental designs. D-optimal design is shown its effectiveness by constructing a reliable model with few experiment setups. The number of required experiments is about twice the number of polynomial coefficients. To construct the model for the velocity, depending on our resource limitations, we decided to select 37 experiments by D-optimal design method. The ranges of each design variable are listed in Table 2.

\begin{tabular}{ccccccc}
\hline & \multicolumn{9}{c}{ Design variables } \\
\cline { 2 - 7 } Name & vol & freq & $d_{1}$ & $d_{3}$ & $w_{1}$ & $w_{3}$ \\
\hline Level & $\{10,12,14,16\}$ & $\{500,1000,5000,7000,10000,20000\}$ & $\{180,540\}$ & $\{180,540\}$ & $\{5,40\}$ & $\{5,40\}$ \\
Coded & $\{-1,-0.33,0.33,1\}$ & $\{-1,-0.6,-0.2,0.2,0.6,1\}$ & $\{-1,1\}$ & $\{-1,1\}$ & $\{-1,1\}$ & $\{-1,1\}$ \\
\hline
\end{tabular}

Table 2: Design variables ranges

It should be noted that using Full factorial design as an alternative of the D-optimal design, it would be needed for the total of $2^{8}$ test setup. Obviously this needs a huge number of simulation run leads to computational expenses.

Using the above information, we are able to fit a reliable model (1) and perform the optimization for maximizing the velocity.

To ensure the accuracy of the fitted model (the goodness of fit), the approximation model is evaluated based on coefficient of determination statistic $\left(R^{2}\right)$, adjusted R-squared statistic adj $-R^{2}$ and root mean square error (RMSE). We also calculate variance inflation factor (VIF) to detect the multicolinearity.

\section{Results and analysis}

Using the information in Table 2, we conduct the experiments with different plasma configurations. The velocity fields generated are recorded using Particle Imaging Velocimetry (PIV). Table 3 summarizes the value of velocity measured for each setting of the sample data. The quadratic polynomial model is then approximated as

$$
\begin{aligned}
\text { Velocity }=- & 1.14-0.00129 x_{1}-0.0019 x_{3}+0.000068 x_{5} \\
& +0.145 x_{6}-0.000007 x_{1} x_{3}+0.000106 x_{1} x_{6}+0.00078 x_{3} x_{6}
\end{aligned}
$$

where $\mathrm{X}=\left(d_{1}, d_{3}, w_{1}, w_{3}, f r e q, v o l\right)$. As can be seen, the model only contains interaction term with the absence of the depth and width of electrode 3. This only is because of the fact that we have an irregular design space which leads to the correlation between the specifications of electrode 1 and 3 . 


\begin{tabular}{|c|c|c|c|c|c|c|c|}
\hline \multirow[b]{2}{*}{ Run } & \multicolumn{6}{|c|}{ Design variables } & \multirow{2}{*}{$\begin{array}{l}\text { response } \\
\text { Velocity }\end{array}$} \\
\hline & vol & freq & $d_{1}$ & $d_{3}$ & $w_{1}$ & $w_{3}$ & \\
\hline 1 & 0.33 & -0.6 & -1 & 1 & 1 & -1 & 1.75 \\
\hline 2 & -0.33 & -1 & -1 & -1 & 1 & 1 & 0.32 \\
\hline 3 & -0.33 & -0.2 & -1 & -1 & 1 & 1 & 0.9 \\
\hline 4 & 0.33 & -0.6 & -1 & -1 & 1 & 1 & 1.67 \\
\hline 5 & -0.33 & 0.2 & 1 & -1 & -1 & 1 & 1.07 \\
\hline 6 & -0.33 & 0.2 & 1 & 1 & -1 & -1 & 0.92 \\
\hline 7 & -0.33 & 0.2 & -1 & 1 & 1 & -1 & 1.46 \\
\hline 8 & -0.33 & 0.2 & -1 & -1 & 1 & 1 & 1.09 \\
\hline 9 & -0.33 & -0.2 & 1 & -1 & -1 & 1 & 0.95 \\
\hline 10 & -0.33 & 0.6 & -1 & 1 & 1 & -1 & 0.81 \\
\hline 11 & -0.33 & 1 & -1 & 1 & 1 & -1 & 1.197 \\
\hline 12 & -0.33 & 0.6 & -1 & -1 & 1 & 1 & 0.56 \\
\hline 13 & 0.33 & -0.6 & 1 & -1 & -1 & 1 & 1.55 \\
\hline 14 & -0.33 & 0.6 & 1 & 1 & -1 & -1 & 0.7 \\
\hline 15 & -0.33 & 0.6 & 1 & -1 & -1 & 1 & 0.64 \\
\hline 16 & -0.33 & -0.2 & 1 & 1 & -1 & -1 & 0.878 \\
\hline 17 & 0.33 & -0.6 & 1 & 1 & -1 & -1 & 1.75 \\
\hline 18 & 0.33 & -0.6 & 1 & -1 & -1 & 1 & 1.81 \\
\hline 19 & -0.33 & -0.6 & -1 & -1 & 1 & 1 & 1.37 \\
\hline 20 & -0.33 & -1 & 1 & -1 & -1 & 1 & 0.45 \\
\hline 21 & -0.33 & -0.2 & -1 & 1 & 1 & -1 & 1.386 \\
\hline 22 & 0.33 & -0.6 & 1 & -1 & -1 & 1 & 1.81 \\
\hline 23 & -0.33 & -1 & -1 & 1 & 1 & -1 & 0.47 \\
\hline 24 & -0.33 & 1 & 1 & -1 & -1 & 1 & 1.02 \\
\hline 25 & -0.33 & -0.6 & -1 & -1 & 1 & 1 & 1.37 \\
\hline 26 & 1 & -0.6 & 1 & -1 & -1 & 1 & 1.95 \\
\hline 27 & -0.33 & -0.2 & 1 & 1 & -1 & -1 & 0.88 \\
\hline 28 & -0.33 & -1 & 1 & 1 & -1 & -1 & 0.435 \\
\hline 29 & -1 & -0.6 & -1 & -1 & 1 & 1 & 1.027 \\
\hline 30 & -1 & -0.6 & -1 & 1 & 1 & -1 & 1.1 \\
\hline 31 & -1 & -0.6 & 1 & 1 & -1 & -1 & 1.046 \\
\hline 32 & -1 & -0.6 & 1 & -1 & -1 & 1 & 1.02 \\
\hline 33 & -0.33 & -0.6 & 1 & -1 & -1 & 1 & 1.08 \\
\hline 34 & 0.33 & -0.6 & -1 & 1 & 1 & -1 & 1.82 \\
\hline 35 & -0.33 & -0.6 & 1 & 1 & -1 & -1 & 1.1 \\
\hline 36 & 1 & -0.6 & 1 & 1 & -1 & -1 & 2.17 \\
\hline 37 & -0.33 & -0.6 & -1 & 1 & 1 & -1 & 1.66 \\
\hline
\end{tabular}

Table 3: Sample data for D-Optimal design (coded) 
The accuracy measurements statistics are shown in Table 4 indicating a reasonable approximation. In addition, the normal probability plot of the residuals shows that the residuals scatter normally (Figure 2). That is, the model (2) is fit reasonably to the experimental data.

\begin{tabular}{ccccc}
\hline & \multicolumn{4}{c}{ Static } \\
\cline { 2 - 5 } Name & $R^{2}$ & Adj $R^{2}$ & RMSE & p-value \\
\hline Value & $95.8 \%$ & $94 \%$ & $11.2 \%$ & 0.002 \\
\hline
\end{tabular}

Table 4: Goodness of fit statistic
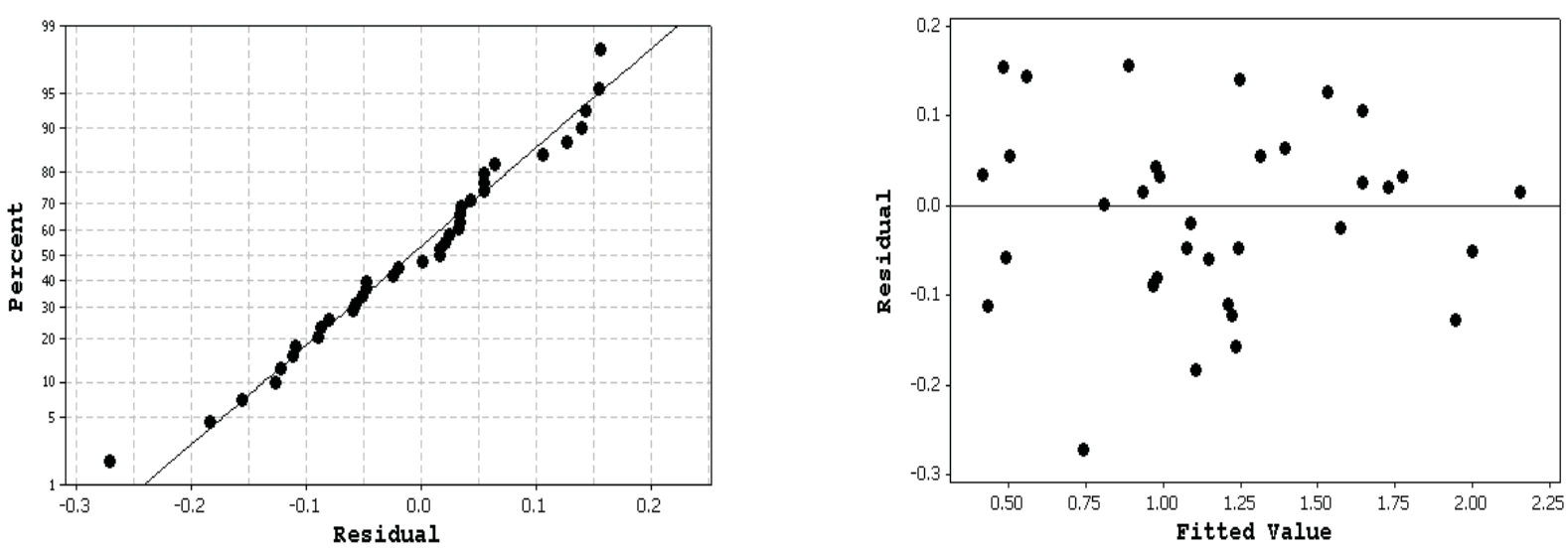

Figure 2: Normal probability plot (left), fitted values versus residual (right)

It should be noted that some of the parameters of the problems are highly correlated with high VIF. Therefore, they were removed in regression analysis for the sake of accuracy of the measurement.

For further investigation, error analysis has been done on the model defined by the difference between the actual response and fitted value. The maximum and average absolute value of the error is presented in Table 5 .

\begin{tabular}{lcc}
\hline & \multicolumn{2}{c}{ Error Analysis } \\
\cline { 2 - 3 } Name & $\operatorname{Max} \mid \%$ error $\mid$ & Average $\mid \%$ error $\mid$ \\
\hline Value & $27.2 \%$ & $8 \%$ \\
\hline
\end{tabular}

Table 5: Error analysis

Using the model of Eq. 1 and the boundary constraints listed in Table 2, the following optimization is formulated:

$$
\begin{aligned}
\text { Maximize } & \text { Velocity }=f\left(\text { volt }, \text { freq }, d_{1}, d_{3}, w_{1}, w_{3}\right), \\
\text { s.t. } \quad & d_{1}<d_{2}<d_{3} \quad \text { or } \quad d_{3}<d_{2}<d_{1}, \\
& 180 \leq d_{1}, d_{3} \leq 540 \\
& 5 \leq w_{1}, w_{3} \leq 40 \\
& 10 \leq \text { vol } \leq 16 \\
& 500 \leq \text { freq } \leq 20000,
\end{aligned}
$$

where the first set of constraints ensures the prescribed distance between the electrodes.

$$
6 \text { of } 9
$$




\begin{tabular}{cccccccc}
\hline & \multicolumn{3}{c}{ Optimum setting } & \multicolumn{3}{c}{ Optimum response } \\
\cline { 2 - 8 } vol & freq & $d_{1}$ & $d_{3}$ & $w_{1}$ & $w_{3}$ & Predicted Velocity & Error interval \\
\hline 16 & 19800 & 536 & 188 & 39 & 16 & 3.02 & $(2.76,3.02,3.27)$ \\
\hline
\end{tabular}

Table 6: Optimum configuration setting

Using MATLAB, we solved the above problem and the result is tabulated in Table 6 .

Figure 3, shows the velocity respect to voltage and depth of electrode 1 as two of the most influenced variables while the other parameters are kept fixed at their optimum value.

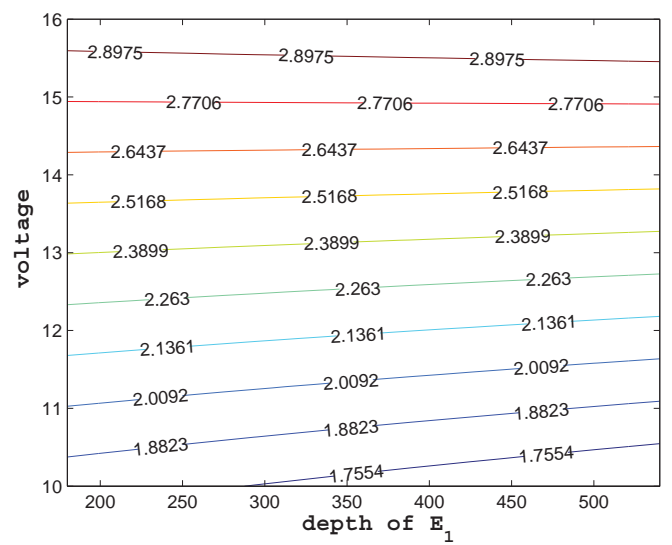

(a) Velocity contour plot

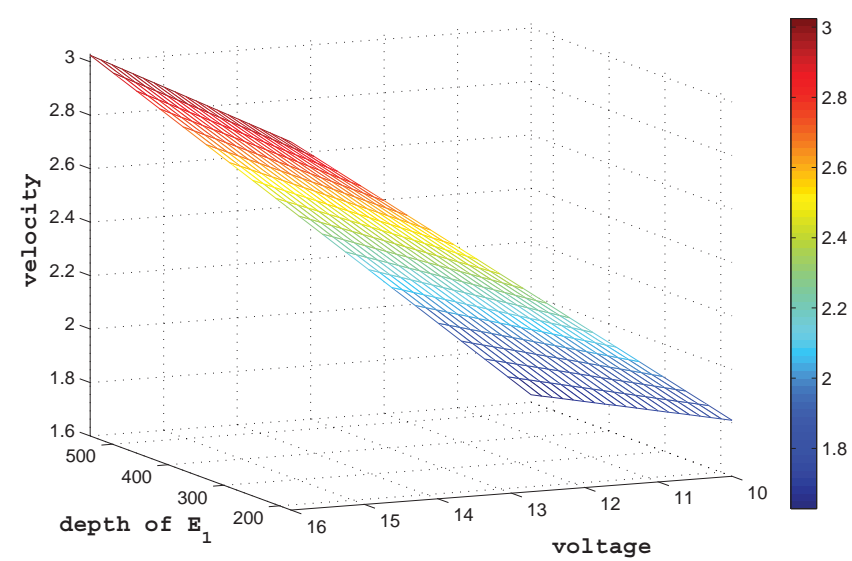

(b) Velocity versus voltage and depth of $E_{1}$

Figure 3: Velocity surface respect to the design parameters

Based on the figure, it is obvious that the surface of velocity respect to voltage and depth of electrode 1, is twisted as a result of interaction term in Eq. 2. The contour plot also shows that with high value of voltage the velocity increases if the electrode 1 is kept deep in the dielectric and away from the exposed electrode.

As can be seen, the error interval (calculating from the average absolute error) contains the velocity which has been verified by our experiments. This shows that the results from the experiments and the numerical optimization provide pretty much the same configuration.

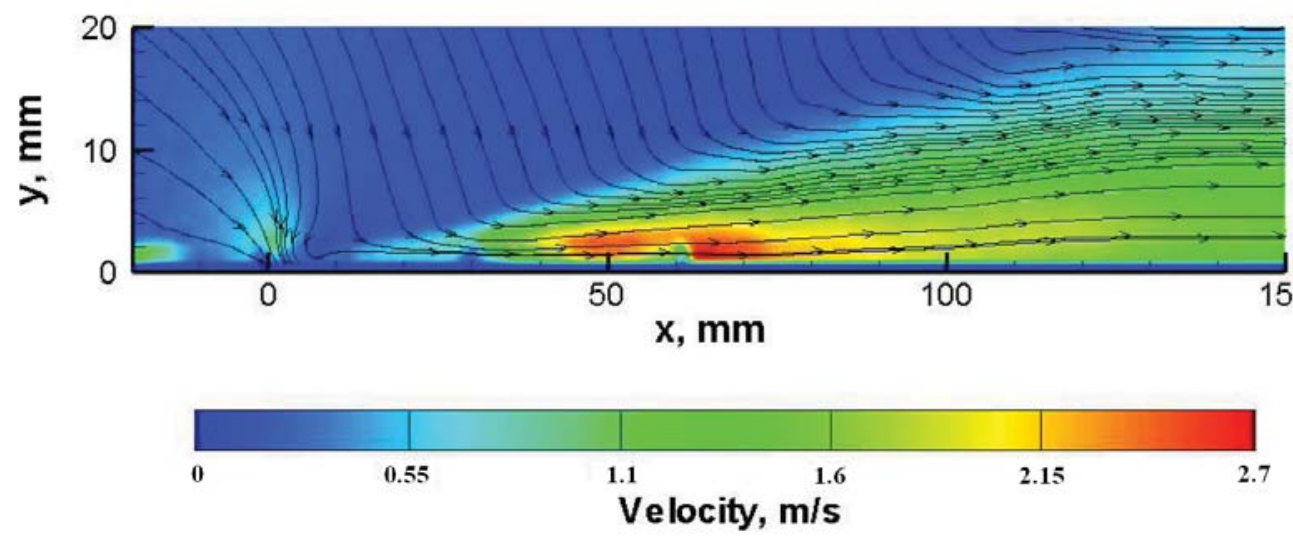

Figure 4: Experimental setup for multiple encapsulated actuator with contour of highest velocity 


\section{Conclusion and future work}

In this paper, we proposed a methodology to study the velocity of the airflow using the Plasma actuator. We have used the optimization technique accompanying by response surface methodology. The D-Optimal experimental design is used to keep the dimensionality of the design space and make the experiments as affordable as possible. After fitting the data to a quadratic polynomial velocity, we maximized the value subject to the problem constraints. We were able to validate the proposed configuration by experimental test data. However, we recognized some correlation between the depth of electrode 1 and 3 and also their width. This is predictable as we face an irregular design shape. In addition, the fitted approximation model only contains the interaction term. For this purpose for further investigation we may consider middle point for these four design parameters to provide a more accurate approximation. As another future consideration, it is worth looking simultaneously at the velocity and the power. This leads to multiobjective optimization and finding the best trade-off for the configurations. ${ }^{38}$ This allows the researcher to find the best possible configuration in order to maximize the airflow meanwhile keeping the power minimum.

\section{Acknowledgments}

The authors would like to thank the school of MACE at the University of Manchester for the financial support. The help and support of the administrative and technical staff of the University of Manchester are also greatly acknowledged.

\section{References}

\footnotetext{
${ }^{1}$ Porter, C., McLaughlin, T., Enloe, C., Font, G., Roney, J., and Baughn, J., "Boundary Layer Control Using a DBD Plasma Actuator,'," AIAA Paper, Vol. 786, 2007, pp. 2007.

${ }^{2}$ Roupassov, D., Zavialov, I., Starikovskii, A., et al., "Boundary layer separation plasma control using low-temperature non-equilibrium plasma of gas discharge," 2006.

${ }^{3}$ Boxx, I., Rivir, R., Newcamp, J., and Woods, N., "Reattachment of a Separated Boundary Layer on a Flat Plate in a Highly Adverse Pressure Gradient Using a Plasma Actuator," 3 rd AIAA Flow Control Conference; San Francisco, CA, American Institute of Aeronautics and Astronautics, 1801 Alexander Bell Drive, Suite 500, Reston, VA, 20191-4344, USA,, 2006.

${ }^{4}$ Jacob, J., Rivir, R., Carter, C., and Estevadeordal, J., "Boundary layer flow control using AC discharge plasma actuators," AIAA 2nd Flow Control Meeting, AIAA Paper, Vol. 2128, 2004.

${ }^{5}$ Font, G., "Boundary layer control with atmospheric plasma discharges," AIAA journal, Vol. 44, No. 7, 2006, pp. 15721578.

${ }^{6}$ Post, M. and Corke, T., "Separation control on high angle of attack airfoil using plasma actuators," AIAA journal, Vol. 42, No. 11, 2004

${ }^{7}$ Huang, J., Corke, T., and Thomas, F., "Plasma actuators for separation control of low-pressure turbine blades," AIAA journal, Vol. 44, No. 1, 2006, pp. 51-57.

${ }^{8}$ Grundmann, S. and Tropea, C., "Delay of Boundary-Layer Transition Using Plasma Actuators," 46 th AIAA Aerospace Sciences Meeting and Exhibit, American Institute of Aeronautics and Astronautics, 1801 Alexander Bell Drive, Suite 500, Reston, VA, 20191-4344, USA, 2008.

${ }^{9}$ Corke, T., He, C., and Patel, M., "Plasma flaps and slats: an application of weakly-ionized plasma actuators," AIAA paper, Vol. 2127, 2004, pp. 2004.

${ }^{10}$ Post, M. and Corke, T., "Separation control using plasma actuators: dynamic stall vortex control on oscillating airfoil," AIAA journal, Vol. 44, No. 12, 2006, pp. 3125.

${ }^{11}$ Asghar, A. and Jumper, E., "Phase synchronization of vortex shedding from two side-by-side circular cylinders using plasma actuators," 42 nd AIAA Aerospace Sciences Meeting and Exhibit, 2004.

${ }^{12}$ McLaughlin, T., Felker, B., Avery, J., and Enloe, C., "Further experiments in cylinder wake modification with dielectric barrier discharge forcing," AIAA paper, Vol. 1409, 2006.

${ }^{13}$ McLaughlin, T., Munska, M., Vaeth, J., Dauwalter, T., Goode, J., and Siegal, S., "Plasma-based actuators for cylinder wake vortex control," AIAA paper, Vol. 2129, 2004

${ }^{14}$ Shunsuke Yamada, Koui Shibata., H. I. S. H. and Motosuke, M., "Flow Behavior behind a Circular Cylinder by DBD Plasma Actuators in Low Reynolds Number," AIAA Paper, 2010.

${ }^{15}$ Thomas, F., Kozlov, A., and Corke, T., "Plasma actuators for cylinder flow control and noise reduction," AIAA J, Vol. 46, No. 8, 2008, pp. 1921-1931.

${ }^{16}$ Enloe, C., McLaughlin, T., VanDyken, R., Kachner, K., Jumper, E., and Corke, T., "Mechanisms and responses of a single dielectric barrier plasma actuator: plasma morphology," AIAA Journal, Vol. 42, No. 3, 2004, pp. 589-594.

${ }^{17}$ Enloe, C., McHarg, M., and McLaughlin, T., "Time-correlated force production measurements of the dielectric barrier discharge plasma aerodynamic actuator," Journal of applied physics, Vol. 103, 2008, pp. 073302.

${ }^{18}$ Enloe, C., McLaughlin, T., VanDyken, R., Kachner, K., Jumper, E., Corke, T., Post, M., and Haddad, O., "Mechanisms and responses of a single dielectric barrier plasma actuator: geometric effects," AIAA journal, Vol. 42, No. 3, 2004 , pp. 595-604.
} 
${ }^{19}$ Roth, J., Sherman, D., and Wilkinson, S., "Electrohydrodynamic flow control with a glow-discharge surface plasma," AIAA journal, Vol. 38, No. 7, 2000, pp. 1166-1172.

${ }^{20}$ Roth, J., Sherman, D., and Wilkinson, S., "Boundary layer flow control with a one atmosphere uniform glow discharge surface plasma," Tech. rep., 1998.

${ }^{21}$ Roth, J., Sin, H., Madhan, R., and Wilkinson, S., "Flow re-attachment and acceleration by paraelectric and peristaltic electrohydrodynamic (EHD) effects," AIAA paper, Vol. 351, 2003, pp. 6-9.

${ }^{22}$ Roth, J. and Dai, X., "Optimization of the aerodynamic plasma actuator as an electrohydrodynamic (EHD) electrical device," AIAA paper, Vol. 1203, 2006, pp. 2006.

${ }^{23}$ Hale, C., Erfani, R., and Kontis, K., "Increasing the Induced Velocity of Dielectric Barrier Discharge Plasma Actuators," CEAS 2009 European Air and Space Conference, 2009.

${ }^{24}$ Hale, C., Erfani, R., and Kontis, K., "Plasma Actuators with Multiple Encapsulated Electrodes to Influence the Induced Velocity," No. 2010-1223 in AIAA, 2010.

${ }^{25}$ Hale, C., Erfani, R., and Kontis, K., "Plasma Actuators with Multiple Encapsulated Electrodes to Influence the Induced Velocity : Further Configurations," No. 2010 in AIAA, 2010.

${ }^{26}$ Queipo, N., Haftka, R., Shyy, W., Goel, T., Vaidyanathan, R., and Kevin Tucker, P., "Surrogate-based analysis and optimization," Progress in Aerospace Sciences, Vol. 41, No. 1, 2005, pp. 1-28.

${ }^{27}$ Myers, R. and Montgomery, D., Response surface methodology, Allyn and Bacon Boston, 1971.

${ }^{28}$ GEP, B. and Draper, N., Empirical model-building and response surfaces, John Wiley and Sons, New York, USA, 1987.

${ }^{29}$ Agatonovic-Kustrin, S., Zecevic, M., Zivanovic, L., and Tucker, I., "Application of neural networks for response surface modeling in HPLC optimization," Analytica chimica acta, Vol. 364, No. 1-3, 1998, pp. 265-273.

${ }^{30}$ Negnevitsky, M., Artificial intelligence: A guide to intelligent systems, Addison-Wesley Longman, 2005.

${ }^{31}$ Goel, T., Haftka, R., Shyy, W., and Queipo, N., "Ensemble of surrogates," Structural and Multidisciplinary Optimization, Vol. 33, No. 3, 2007, pp. 199-216.

${ }^{32}$ Mack, Y., Goel, T., Shyy, W., Haftka, R., and Queipo, N., "Multiple surrogates for the shape optimization of bluff bodyfacilitated mixing," Proceedings of the 43rd AIAA aerospace sciences meeting and exhibit, Reno, NV, 2005, pp. 2005-0333.

${ }^{33}$ Giunta, A., Balabanov, V., Haim, D., Grossman, B., Mason, W., Watson, L., and Haftka, R., "Wing design for a high-speed civil transport using a design of experiments methodology," Proceedings of the 6th AIAA/USAF/NASA/ISSMO Symposium on Multidisciplinary Analysis and Optimization, pp. 168-183.

${ }^{34}$ Vladimir, B., Dan, H., and Bernard, G., "Wing Design for a High-Speed Civil Transport Using a Design of Experiments Methodology," 1996.

${ }^{35}$ Jansson, T., Nilsson, L., and Redhe, M., "Using surrogate models and response surfaces in structural optimization-with application to crashworthiness design and sheet metal forming," Structural and Multidisciplinary Optimization, Vol. 25, No. 2, 2003, pp. 129-140.

${ }^{36}$ Samad, A., Kim, K., Goel, T., Haftka, R., and Shyy, W., "Multiple surrogate modeling for axial compressor blade shape optimization," Journal of Propulsion and Power, Vol. 24, No. 2, 2008, pp. 302-310.

${ }^{37}$ Simpson, T., Mauery, T., Korte, J., and Mistree, F., "Kriging models for global approximation in simulation-based multidisciplinary design optimization," AIAA journal, Vol. 39, No. 12, 2001, pp. 2233-2241.

${ }^{38}$ Erfani, T. and Utyuzhnikov, S., "Directed Search Domain: A Method for Even Generation of Pareto Frontier in Multiobjective Optimization," Engineering Optimization, 2010. 